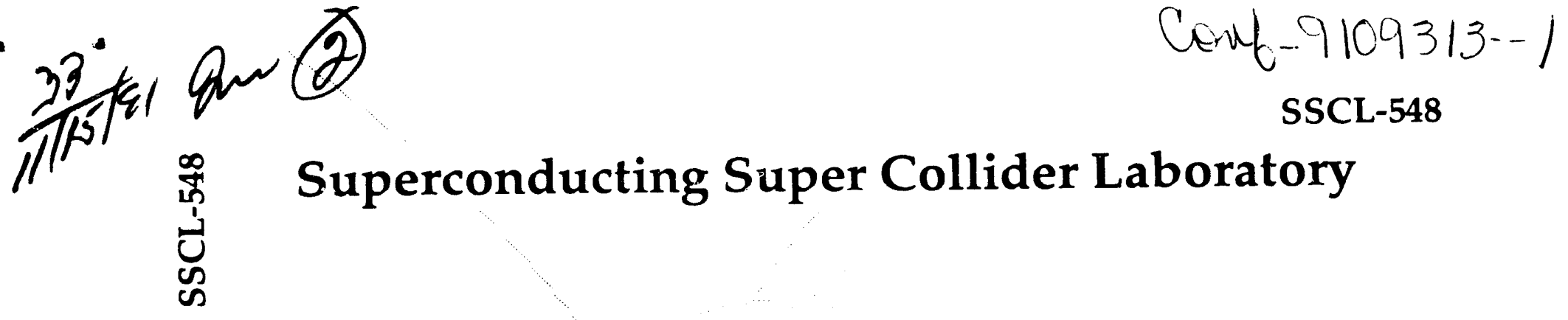

\title{
A Brief Introduction to the Strong CP Problem
}

Dan-di Wu

September 1991 


\title{
A Brief Introduction to the Strong CP Problem*
}

\author{
Dan-di Wu \\ Physics Research Division \\ Superconducting Super Collider Laboratory ${ }^{\dagger}$ \\ 2550 Beckleymeade Ave. \\ Dallas, TX 75237 \\ and \\ School of Physics \\ University of Melbourne \\ Parkville, Victoria 3052, Australia
}

September 1991

\section{DISCLAIMER}

\begin{abstract}
This report was prepared as an account of work sponsored by an agency of the United States Government. Neither the United States Government nor any agency thereof, nor any of their employees, makes any warranty, express or implied, or assumes any legal liability or responsibility for the accuracy, completeness, or usefulness of any information, apparatus, product, or process disclosed, or represents that its use would not infringe privately owned rights. Reference herein to any specific commercial product, process, or service by trade name, trademark, manufacturer, or otherwise does not necessarily constitute or imply its andorsement, recommendation, or favoring by the United States Government or any agency thereof. The views and opinions of authors expressed herein do not necessarily state or reflect those of the United States Government or any agency thereof.
\end{abstract}

- Presented at the Workshop in Honor of F.C.C. Sudarshan's Contritutions in Theoretical Physics, September 1991

† Operated by the Universities Research Association. Inc., for the C.S. Department of Energy under Contract No. DE-AC $35-89$ ER $404 \times 6$

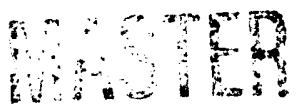




\title{
A Brief Introduction to the Strong CP Problem
}

\author{
Dan-di Wu
}

\begin{abstract}
The present status of the strong CP problem is briefly reviewed in a heuristic way. A crisis in EDMN calculation is explained. The equation of vacuum alignment obtained by the author and collaborators last year put a constrairt on strong CP parameters. Thus the strong CP will be forced to vanish in one of the three scenarios characterized by axion, zero quark mass, and vanishing quark condensate.
\end{abstract}


I shall try to explain briefly the strong CP problem and its possible solutions.

The strong CP problem is a serious flaw of the standard model (SM), especially of its strong interaction section. This problem has been attacked for 15 years. Some solutions have been found, but none of them is conclusive. If it turns out that none of them works, it might mean that there is a deep defect in our basic understanding of SM.

The electroweak section of SM with three generations of quarks may explain the observed CP-violating process $K_{L} \rightarrow 2 \pi$ very well. The essential quantity that appears in the calculation is the rephasing (vector-like) invariant ${ }^{1}$ of the Kobayashi-Maskawa matrix:

$$
t=S_{1}^{2} S_{2} S_{3} C_{1} C_{2} C_{3} \sin \delta
$$

If the decay width of the kaon is proportional to $S_{1}^{2}$, then the expected CP-violating rate $\epsilon=\left(K_{L} \rightarrow 2 \pi\right) /\left(K_{S} \rightarrow 2 \pi\right)$ is about $S_{2} S_{3} C_{1} C_{2} C_{3} \sin \delta$. According to the present knowledge collected from other experiments, this value is about $10^{-3}$, compared with the experimental value of $\epsilon=2.7 \times 10^{-3}$. It is remarkable that the correct order of magnitude of $\epsilon$ can be obtained so easily.

In contrast to this success of the electroweak theory, the possible strong CP-violating effects-such as the electric dipole moment of the neutron (EDMN), described by the allowed parameter $\bar{\theta}$ (to be defined later)--have been ruled out to a very high precision. This requires $\bar{\theta}$ to be extremely small. The question of why $\bar{\theta}$ should be so small is studied under the title "Strong CP Problem."

Limited by space, let us concentrate on a QCD model with only one quark. The mass term of the fermion is usually written as $-6 m \psi$. Since QCD is part of SM and there is CP violation in SM anyway, the following mass term is in general allowed:

$$
\mathcal{L}_{m}=-\psi \tilde{m} \psi
$$

where $i \bar{n}=m e^{i \gamma_{5} \phi}$ is called the fermion mass with a chiral phase, or sometimes, the complex mass. Please be careful not to confuse $\tilde{m}$ with the effective complex mass of a decaying particle, $m+i-/ 2$. The 75 part of Eq. (2) is $P$ - and T-odd and is hermitian. The intrusion of the new parameter o did not cause attention intil the importance of another term in the pure gauge part of the QC'D Lagrangian was noticed.2.3 This term is called the $\theta$-term:

$$
\dot{\mathcal{L}}_{\theta}=H G_{\dot{r}} \ddot{G}
$$


where

$$
G^{\prime} \tilde{G}^{\prime}=\frac{1}{32 \pi^{2}} \varepsilon_{\mu \nu \varrho \sigma} G_{a}^{\mu \nu} G_{a}^{\varrho \sigma}
$$

This term is also $\mathrm{P}$ - and T-odd. Furthermore, the two terms are related by chiral rotation due to the triangle anomaly. ${ }^{4}$ That is, when

$$
\psi \longrightarrow \psi^{\prime}=e^{i \alpha \gamma_{5} / 2} \psi,
$$

we obtain

$$
\phi \longrightarrow \phi^{\prime}=\phi+\alpha, \quad \theta \longrightarrow \theta^{\prime}=\theta-\alpha
$$

Note that

$$
\ddot{\theta}=\theta+\phi
$$

will not be changed under chiral rotations. Therefore, it is impossible to "turn away" CP violation terms by a chiral rotation once $\bar{\theta}$ is fixed. Besides, chiral rotation is not the symmetry of the system. The general equivalence of the different Lagrangian related by chiral rotation is under question, unless a corresponding adjustment of vacuum is made (see later).

The question of what physical effects could be due to the above strong CP violation is somehow subtle. Because the strong CP-violating terms do not change the flavors of the quarks as the weak interaction does, then the strong C.P violation will certainly not be the leading effect in weak decays. Attention has been focused on the process $\eta \rightarrow 2 \pi$ and on EDMN: both need P- and T-violation to happen. Since ED.MN has been experimentally narrowed down to a very small number, it was claimed that the measured bonnd on EDMN placed a stringent bound on the value of $\ddot{\theta}$. Howerer, the celebrated calculation to relate EDMN to $\theta$ by C'rewther et al. ${ }^{5}$ (CDVW) has recently been criticized by Banerjee, Chattergee, and Mitra ${ }^{6}$ and by Gupta, Mckellar, and Wu. The method of CDVW can be sketched as follows. They first shift $\bar{\theta}$ to the mass term. so that superficially all strong CP comes from the mass term. They then use the chiral perturbation theory the current algebra) to calculate EDW in terms of $\theta$. In doing so, the possible complex matss of the neutron caused by the complex mass of the constituent quarks has not been consistently handled. Putting it succinctly. there is a risk that the final result would take the following form:

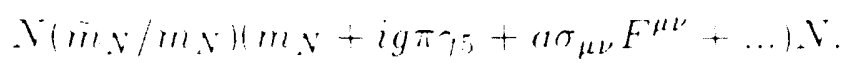

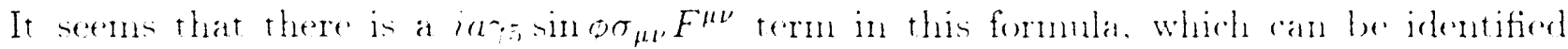

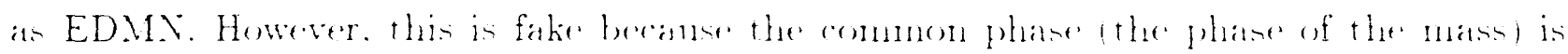


protected by a perturbative symmetry of the original QCD-effective Lagrangian. This phase will disappear if a suitable wave function of the neutron is chosen which satisfies the Dirac equation with a complex neutron mass. Therefore, unless non-perturbative effects are explicitly included, it is impossible to produce a non-zero EDMN.

Though the result of CDVW is criticized, it does not mean that the strong CP effects do not exist. Common wisdom tells us that if we can establish a meaningful relation among some theoretical parameters, such as mass and the strong CP parameters, these parameters must not be redundant ones. They must have some effects. I shall introduce you to such a relation called the equation of vacuum alignment (EVA) established by Huang, Viswanathan, and $\mathrm{Wu}^{8}$ (HVW). If strong $\mathrm{CP}$ does contribute to EDMN, it is expected, from a dimensional argument, that

$$
E D M N \sim e \bar{\theta} / m_{N} \sim 10^{-14} \bar{\theta} e \cdot \mathrm{cm}
$$

To meet the experimental bound. $\bar{\theta}$ has to be extremely small:

$$
\theta \leq 10^{-11}
$$

Now let us discuss the promised equation of acuum alignment. The EVA can be obtained by the use of inwariance of the functional under chiral transformation, as all fermion fields are integrated out in the functional. It reads

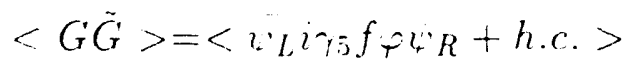

where $r n e^{t 0}$ is replaced by $f_{\mathcal{T}}$. with $\hat{\tau}$ the Higgs field and $f$ the Fukawa coupling constant. Let us specify the vacrum by the following equations:

$$
\left\langle\because>=\left\langle G_{\mu \nu}\right\rangle=0 . \quad\langle\because u R\rangle=\frac{1}{2} C_{d} \neq 0, \quad \tau_{\tau}>=v c^{i a}\right.
$$

with $C_{d}$ the dynamial condenate of the quark. Nege Pong disconsed this quantity in this session. By choosing $C_{d}$ to be real and negative. as perpple nsually do, we actually choose a specifie racumm orientation in the rhimal frane. Cienerally speaking. $C_{d}$ can have an

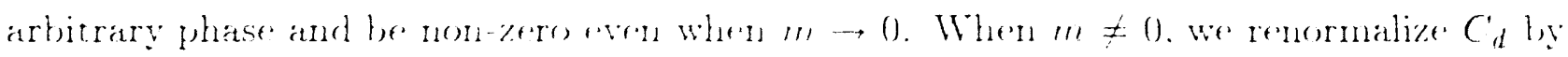


subtracting the contribution due to the current mass. With Eq. (12), Eq. (11) is expressed at the tree level of the Higgs interactions as

$$
<G \tilde{G}>=m C_{d} \sin \phi
$$

where

$$
m=|f| v, \quad f=|f| e^{i \phi_{f}}, \quad \phi=\phi_{f}+a .
$$

Eq. (13) is the EVA of the question. Slightly different equation for light quarks only has been found by CDVW and by 't Hooft ${ }^{9}$ using low-energy effective theories of QCD. Unfortunately, their equation was not seriously considered in the calculation of strong CP effects mentioned previously.

We find from EVA that the values of the phase of the mass $\phi$ are constrained (so is $\theta$ ) if $\bar{\theta}$ is fixed. The strong-interaction dynamics come into play in EVA as represented by $C_{d}$, the dynamical condensate. The vacuum specification of Eq. (12) accompanies EVA and makes it impossible to shift $\bar{\theta}$ arbitrarily without changing the phase of $C_{d}$ at the same time. As we pointed out before, ${ }^{10}$ it is impossible $i$ shift the strong CP completely to the $\theta$-term without changing the condition of $C_{d}$ being real at the same time. Different Lagrangians related by chiral rotations are generally not equivalent unless corresponding rotations of the vacuum are taken into account by changing the phase of $C_{d}$.

EVA also provides three possible scenarios in which $\langle G \tilde{G}\rangle$ is forced to vanish. The first is the famous Pecoi-Quinn (PQ) scenario. ${ }^{3}$ The so-called PQ symmetry makes the phase $a$ of the Higgs field an arbitrary parameter. In this one-quark model the PQ symmetry can be reached by one Higgs field. But when there are quarks with two different electric charges. two Higgs tields are needed to meet the PQ symmetry. One can then always choose a to make $\phi=0$. A consequence of PQ symmetry is the necessity of the ghost particle called axion, ${ }^{12}$ which is a pseudo-scalar particle predicted but not found after an exhaustive ten-year search. "Invisible" axion models have been invented, but they are complicated and unappealing.

The second scenario is $m=0$ (e.g., for the u quark). Since there is no reason why the u quark should not obtain a small mass. this sconario is regarded as unnatural.

The third scenario. newly proposed by HVW. is $C_{d}^{\prime}=0$ (e.g., for a heavy quark - the bor $t$ quark). (of comrse. the t quark should not be too heavy, if we assume it is the one to take the responsibility. Because if $m t$ is too large, it will meet the condition for the $t$ rondensate to form due to the likawa-like interaction. as described by Professor Nambu 
at this conference. The third scenario needs phase transition in dynamical chiral symmetry breakdown, when the current mass of the quark increases to exceed a certain vaiue. While $C_{d}$ for the light quarks must be non-zero as indicated by the success of the current algebra, $C_{d}$ might vanish when the current mass of the quark becomes too heavy. Proof of this phase transition ${ }^{12}$ requires a deep commitment to the strong interaction dynamics. The solution of the strong CP problem (if there is a problem) probably lies in the dynamics of QCD itself if the phase transition does exist. 


\section{ACKNOWLEDGEMENTS}

I thank George for his encouragement and the conference organizer for an instantaneous arrangement of this tall. Comments on the manuscript from B. Grinstein are appreciated. 


\section{REFERENCES}

1. Dan-di Wu, Phys. Rev. D33 (1986) 860; High Energy Phys. Nucl. Phys. 10 (1986) 684 (in Chinese).

2. A. Polyakov, Phys. Lett. 59B (1975) 82; A. Belavin, A. Polyakov, A. Schwartz, and Yu. Tyupkin, ibid (1975) 85; G. 't Hooft, Phys. Rev. Lett. 37 (1976) 8.

3. R. Peccei and H. Quinn, Phys. Rev. Lett. 38 (1977) 1440; Phys. Rev. D16 (1977) 1796.

4. J. Steinberger, Phys. Rev. 76 (1949) 1180; S. Adler, Phys. Rev. 177 (1969) 2426; J. Bell and R. Jackiw, Nov. Cim. 60 (1969) 47.

5. R. Crewther, P. Di Vicchia, G. Veneziano, and E. Witten, Phys. Lett. 88B (1979) 123.

6. H. Banerjee, D. Chatterjee, and P. Mitra, Saha Institute Preprint, SINP-TNP-90/5; SINP-TNP-90/17. See also A. Abada, J. Galand, A. Le Yaouanc, L. Oliver, O. Pene, and J.-C. Raynal, Orsay preprint, LPTHE 90/53; Phys. Lett. 256B (1991) 508.

7. V. Gupta, B.J.H. MCKellar, and Dan-di Wu, Melbourne University Preprint, UM-P91/13; UM-P-91/49.

8. Z. Huang, K.S. Viswanathan, and D.D. Wu, Simon Fraser University Preprint, SFUJuly-90-1; Mod. Phys. Lett. A6 (1991) 711; SFU-July-91-4 and UM-P-90/91 to appear in Comm. Theo. Phys.

9. R. Crewther, in Field Theor. Methods in Part. Phys., Nato Advanced Study; P. Di Vicchia and G. Veneziano, Nucl. Phys. B171 (1980) 253; E. Witten. Ann. Phys. (NY) 128 (1980) 363: G. 't Hooft, Phys. Rep. 142 (1986) 357.

10. Z. Huang and D.D. Wu, Simon Fraser University Preprint, SFU-Sep-90-4, to appear in Comm. Theo. Phys.

11. S. Weinberg, Phys. Rev. Lett. 40 (1978) 223.

12. Z. Huang and K.S. Vismanathan. Simon Fraser University Preprint. 

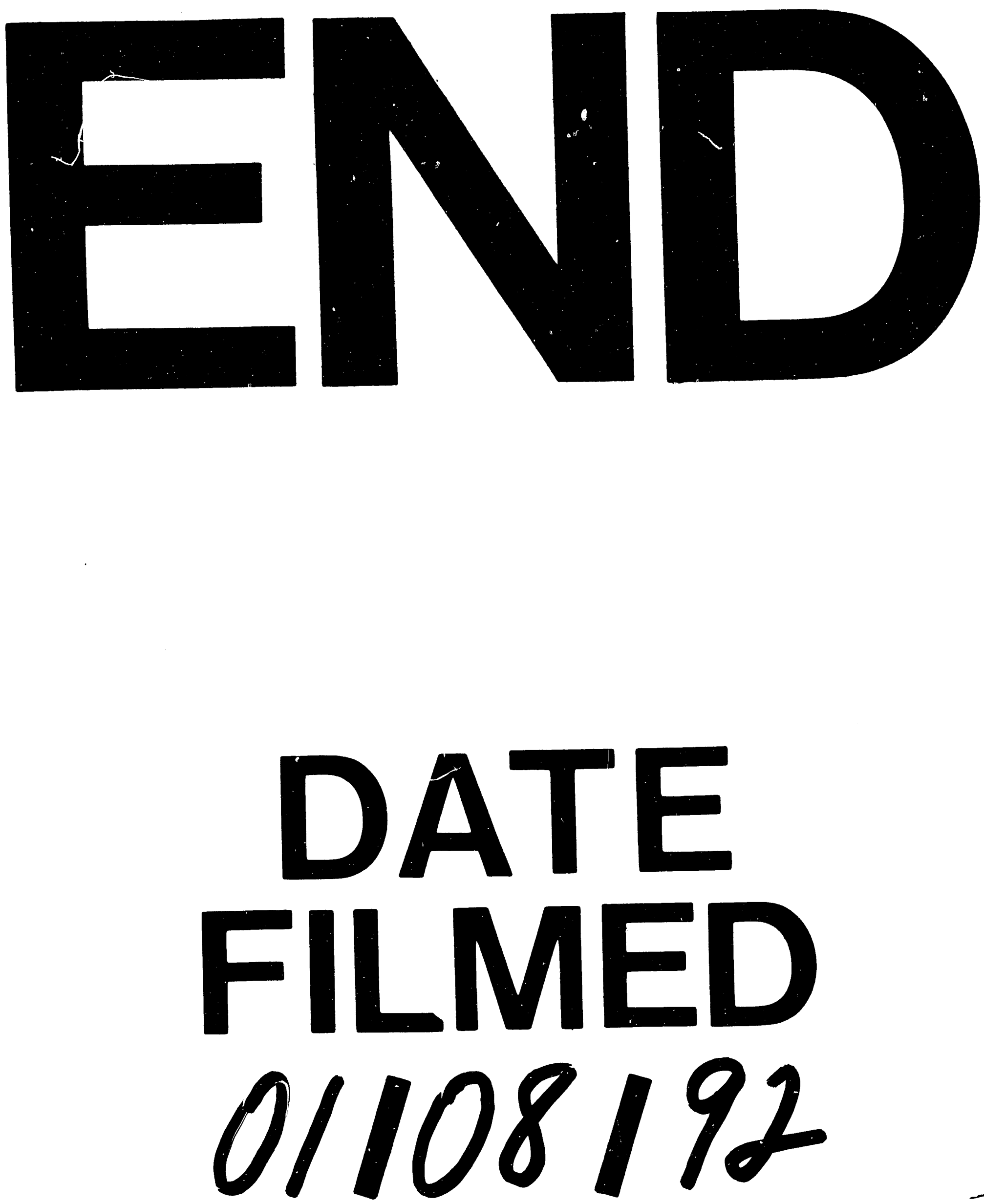
